\title{
Sucesión de un bosque tropical seco en la Isla San Lucas, Puntarenas, Costa Rica
}

\author{
German Vargas G. y Jairo E. Hidalgo-Mora \\ Escuela de Ciencias Biológicas, Universidad Nacional, Heredia, Costa Rica; gevargu@gmail.com, jairohm.9@gmail.com
}

Recibido 15-II-2013 Corregido 18-IV-2013 Aceptado 3-V-2013

\begin{abstract}
Tropical dry forest succession on the San Lucas island, Puntarenas, Costa Rica. The tropical dry forest is the most threatened ecosystem in the neotropics, and management strategies are needed to conserve the last remnants of this type of forest. Using the plant diversity as an indicator, we evaluate the natural regeneration and the successional status of a forest in the Refugio Nacional de Vida Silvestre Isla San Lucas. We established 13 plots where we measured tree diameter, height and crown cover. We also measured the regeneration by counting and identifying all the saplings of the plots. We calculated the basal area, aerial biomass, relative density, relative frequency, relative dominance and the importance value index (IVi) at the family and species level, on both the saplings and adult trees. The island forest presented an average cover of $66,11 \%$ with high variation among plots $( \pm 20,22 \%)$ and it contains 25 species of saplings and trees. About half $(52,2 \%)$ of the tree individuals presented a height between 5,87 and $9,90 \mathrm{~m}$. Also we found a typical J inverted distribution of the diameter classes, which evidenced healthy forest regeneration potential, related with the high thin stem density. Fabaceae and Sapindaceae families presented the highest IVi, for both trees and saplings respectively. A species evenness of $52 \%$ among stand ages groups indicates a species turnover between the trees and the tree saplings. Although the diversity value was pretty similar, tree sapling diversity presented a slightly higher value; a commonly found trait in young secondary forests.
\end{abstract}

\section{KEY WORDS}

Tropical dry forest, natural succession, plant diversity, importance value index, secondary forest

\section{RESUMEN}

El bosque tropical seco (BTS) es actualmente el ecosistema más degradado y fragmentado de todos los tipos de bosque de tierras bajas tropicales; lo que ha generado la necesidad de un manejo adecuado de los últimos remanentes. Evaluamos la sucesión y el grado de regeneración utilizando como indicador la diversidad vegetal de un BTS en el Refugio Nacional de Vida Silvestre Isla San Lucas. Se establecieron 13 parcelas y en cada una se midió el diámetro a la altura del pecho (DAP, 1,30m) de los árboles, su altura; y la cobertura del dosel. Posteriormente se midió el estado de regeneración del ecosistema mediante árboles juveniles (DAP $<10 \mathrm{~cm}$ y altura entre 1 y $3 \mathrm{~m}$ ). Se calculó el área basal, la biomasa aérea, densidad relativa, frecuencia relativa, dominancia relativa y el índice de valor de importancia (IVi), tanto a nivel familia como para cada una de las especies de árboles e individuos jóvenes. El ecosistema presentó una cobertura vegetal de $66,11 \%$ con una alta variación entre sitios $\pm 20,22 \%$, donde se encontraron 25 especies para ambas clases etarias. Para la altura se encontró que un $52 \%$ de los árboles medidos se encontraban entre los 5,87 y 9,90m. La distribución de clases diamétricas en forma de $J$ invertida es un indicativo de un bosque en proceso de regeneración, esto porque hay una mayor densidad de tallos delgados. En el grupo de árboles la familia Fabaceae presentó el IVi más alto, mientras que Sapindaceae en el grupo de los latizales. La similitud entre especies fue de $52 \%$, indicando un cambio de un grupo a otro. La diversidad entre árboles y latizales es similar, con una tendencia mayor hacia los latizales, lo cual es una característica típica de bosques secundarios jóvenes.

PALABRAS CLAVE

Bosque tropical seco, sucesión natural, diversidad vegetal, índice de valor de importancia, bosque secundario
En el Golfo de Nicoya, sitio en donde se encuentra la isla San Lucas, predomina el bosque tropical seco (Bolaños, Watson \& Tosi, 2005). Dicha biota, es un ecosistema de ambientes extremos, el cual en muchas partes del globo esta en transición a desiertos y sabanas (Murphy \& Lugo, 1986). Se caracteriza por presentar una estacionalidad marcada, delimitada por un rango de precipitaciones que van desde 250 a $2000 \mathrm{~mm}$ al año, dependiendo del sitio (Vieira \& Scariot, 2006).

Siendo un ecosistema dominado por árboles deciduos, arbustos y pastizales, en él se pueden encontrar especies vegetales de mucha importancia económica tales como 
Guaiacum sanctum (Zygophyllaceae) y Swietenia humilis (Meliaceae) (Gillespie, Grijalva \& Farris, 2000). Además está presente en suelos con alta disponibilidad de nutrientes, $\mathrm{pH}$ medianamente alto y poco aluminio debido a su origen volcánico, donde los procesos de lixiviación y formación de quelatos son menores (Pennington, Prado $\&$ Pendry, 2000). Motivo por el cual se ha convertido en uno de los ecosistemas más degradados y fragmentados por acción del ser humano (Janzen, 1988; Herrerías-Diego, Quesada, Stoner \& Lobo, 2006). De esta forma, se crea un panorama negativo, ya que dependiendo del grado de alteración que sufra un ecosistema, tendrá una relación directa con la regeneración y las especies que colonicen el sitio, y logren funcionar como atrayentes para especies de animales y dispersores de otras especies (Kennard, Gould, Putz, Fredericksen \& Morales, 2002).

A lo anterior mencionado se agrega el hecho que no todas las especies van a tener la misma respuesta ni las mismas características y estrategias para evadir la competencia y la depredación (Miller \& Kauffman, 1988), teniendo como consecuencia principal que los bosques tropicales secos alterados muy difícilmente van a volver a tener la composición de especies inicial y una estructura similar (Sabogal, 1992). Es por esto que, comprender los procesos de regeneración de este tipo de ecosistema y sus mecanismos de cambio entre estados de sucesión, son de vital importancia para lograr una exitosa restauración del bosque tropical seco (Lebrija-Trejos, Bongers, Pérez-García \& Meave, 2008).

El objetivo de este trabajo fue evaluar la sucesión natural y el grado de restauración de un bosque tropical seco en la isla San Lucas utilizando como indicador la diversidad vegetal.

\section{METODOLOGÍA}

Sitio de estudio: En octubre del 2011 se llevó a cabo el estudio en la isla San Lucas, la cual está situada en la costa pacífica de Costa Rica, en el Golfo de Nicoya $\left(9^{\circ} 56^{\prime} \mathrm{N}\right.$ y $\left.84^{\circ} 54^{\prime} O\right)$. La isla se encuentra en la zona de vida de bosque tropical seco (Bolaños et al., 2005), la cual se caracteriza por tener un ámbito de precipitación media anual que varía entre los 800 y $2100 \mathrm{~mm}$ y ésta se da de forma estacional en una única época del año llamada estación lluviosa y una estación seca que va de noviembre a mayo (Holdridge, 1978).

Caracterización vegetal: En el sitio se establecieron 13 parcelas de $100 \mathrm{~m}^{2}(10 \times 10 \mathrm{~m})$ en diferentes zonas de la isla. En cada una de las parcelas se estructuró el ecosistema midiendo el diámetro a la altura del pecho
(DAP, 1,30cm) de los árboles mayores a $10 \mathrm{~cm}$ de DAP, la altura de los mismos árboles y se estimó la densidad de cobertura del dosel utilizando un densiómetro en cada una de las esquinas de las parcelas y en el centro de las mismas. Posteriormente se procedió a medir el estado de la restauración del ecosistema en nueve de las 13 parcelas, en las cuales se determinó familia y especie de los individuos presentes. Se contaron los árboles con un tallo leñoso menor a $10 \mathrm{~cm}$ de DAP y una altura entre 1 y $3 \mathrm{~m}$.

Análisis de resultados: Se estableció una tabla de distribución de frecuencias para separar categorías diamétricas y alturas, con el fin de estimar el estado sucesional del ecosistema. Para evaluar la composición vegetal, proporciones y las interacciones entre especies de la comunidad, primero se calculó el área basal $(A b)$ y la biomasa aérea $(B a)$ en base a la ecuación desarrollada por (Yepes, del Valle, Jaramillo \& Orrego, 2010) para un bosque secundario. Luego se calculó la densidad relativa, la frecuencia relativa, la dominancia relativa y el índice de valor de importancia (IVi) tanto para familia como para cada una de las especies de acuerdo a lo establecido por (Nebel et al., 2001), utilizando las ecuaciones desarrolladas por Mori, Boom, de Carvalho y dos Santos (1983) y, Curtis y McIntosh (1950, 1951) respectivamente.

La regeneración se analizó de forma similar, exceptuando que el IVi se calculó para individuos jóvenes y no para árboles, el cual se basa en la frecuencia relativa y la densidad relativa. Luego se comparó lo encontrado con hábito arbóreo con las especies de regeneración mediante la diversidad alfa y se realizó una curva de rarefacción para estimar la riqueza de especies. Asimismo se efectuó un índice de dominancia de Simpson y un índice de equidad de Pielou para cada comunidad vegetal. Finalmente se evaluó la diversidad beta mediante un índice de similitud de Sorensen.

\section{RESULTADOS}

Estructura del ecosistema: El ecosistema presentó

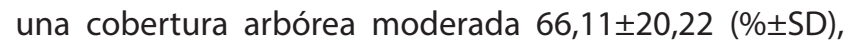
la cual fue variable entre las parcelas de muestreo. Se encontraron un total de 77 árboles pertenecientes a 25 especies distribuidas en 15 familias. De esos árboles un $78 \%$ poseían un DAP entre los 10 y $40 \mathrm{~cm}$, lo que incluiría dos clases diamétricas, donde encontramos la mayor cantidad de individuos, mostrando así la forma característica de $J$ invertida (Fig. 1). Para la altura se encontró que un $52 \%$ de los árboles medidos presentaron una altura entre los 5,87 y 9,90 m (Fig. 1). 
Las familias Fabaceae, Malvaceae, Polygonaceae y Rubiaceae resultaron ser las más importantes dentro del ecosistema, además obtuvieron la mayor área basal y biomasa aérea; las restantes 11 familias poseían valores bajos de área basal, biomasa aérea e índice de valor de importancia (Fig. 2).
Las especies de árboles reflejaron lo observado en los valores de importancia de las familias, aunque varió un poco, ya que la especie con mayor importancia resultó ser Guazuma ulmifolia (Malvaceae), seguida por Gliricidia sepium (Fabaceae) e Inga marginata (Fabaceae), siendo las mismas especies con mayor área basal y biomasa aérea.

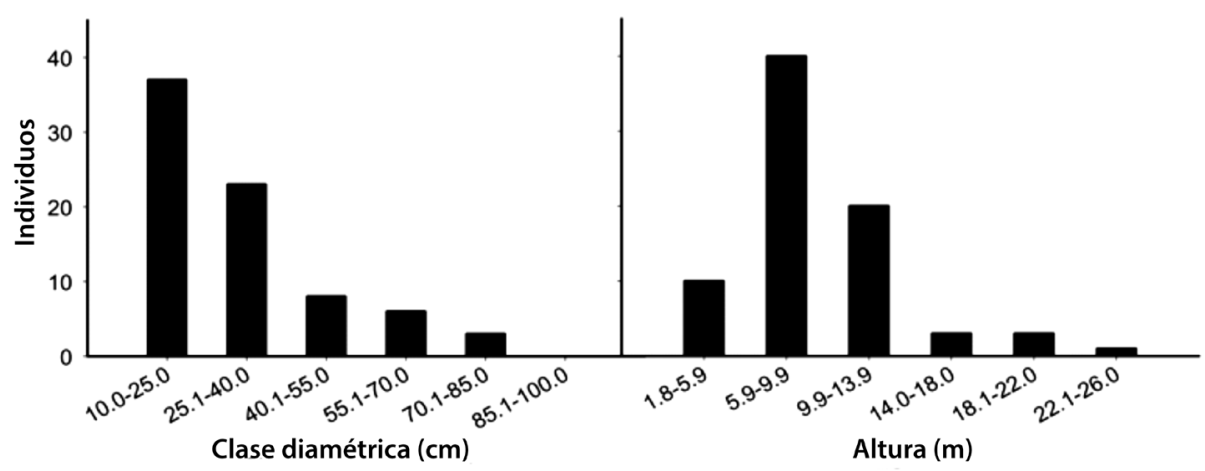

FIG. 1. Categorización de individuos por clases diamétricas y alturas en Isla San Lucas, Puntarenas, Costa Rica.

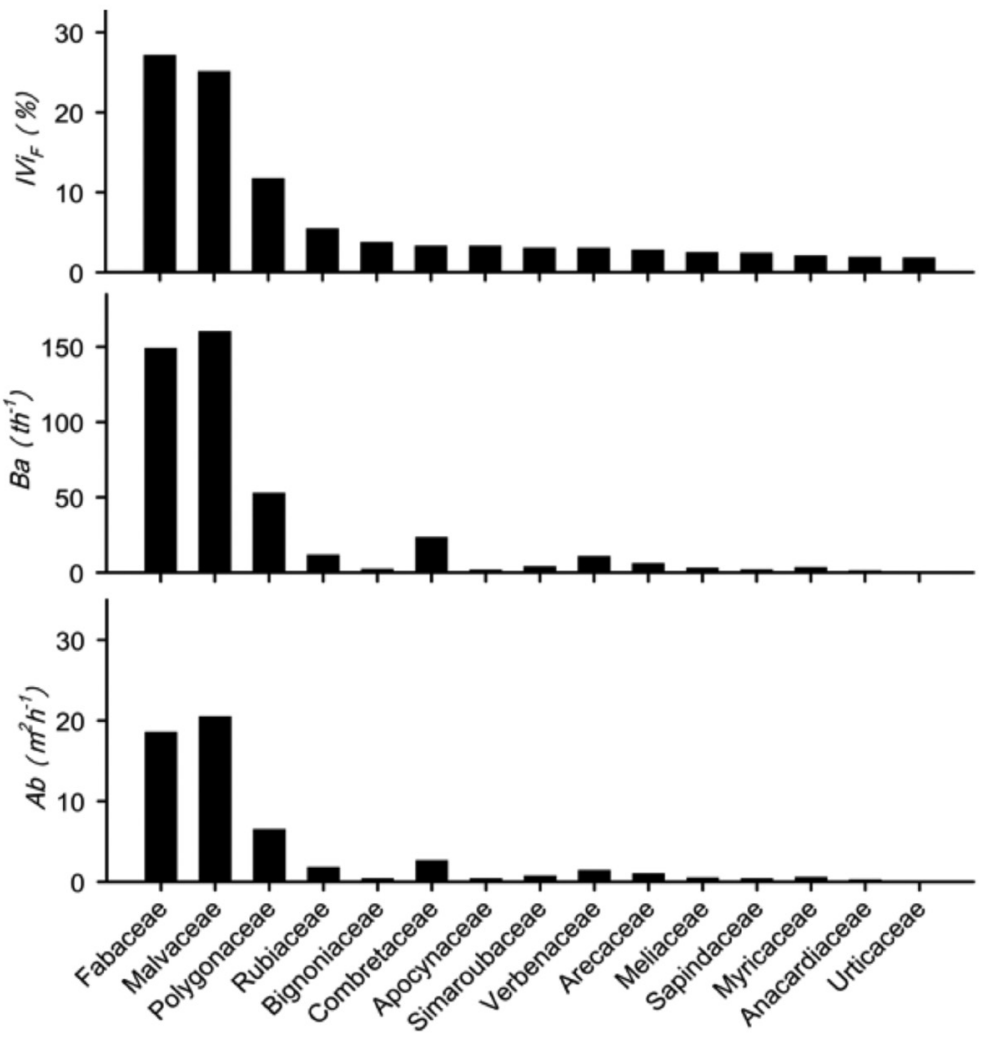

FIG. 2. Índice de valor de importancia (IViF), biomasa aérea (Ba) y área basal (Ab) para cada una de las familias con hábito arbóreo en Isla San Lucas, Puntarenas, Costa Rica. 
No en todos los casos la biomasa aérea resultó correspondiente al índice de valor de importancia, tal es el caso de Terminalia catapa (Combretaceae) (Fig. 3).

Estado de regeneración: En las nueve parcelas en las cuales se estimó la regeneración se contabilizaron 277 individuos distribuidos en 14 familias y en 25 especies. Se encontraron dos familias dominantes las cuales fueron Sapindaceae y Fabaceae (Fig. 4), mientras que las restante 12 familias presentaron valores similares de importancia dentro del ecosistema (Fig. 4).
Para las especies el resultado fue distinto, donde solo Meliccocus bijugatus (Sapindaceae) presentó un $\mathrm{IVi}_{\text {sp }}$ relativamente alto. Evidenciando que no hubo una clara supremacía en cuanto al $I V i_{S p}$ de alguna otra especie (Fig. 5).

Finalmente se observa un cambio en la composición de especies de un estado de sucesión a otro, esto a pesar de que la riqueza de especies se mantiene. La dominancia más alta se presentó en el grupo de latizales. En cuanto a la equitatividad, la comunidad arbórea $(n=77, J=0,85$, $\mathrm{D}=0,10)$ presentó un valor más alto en comparación con

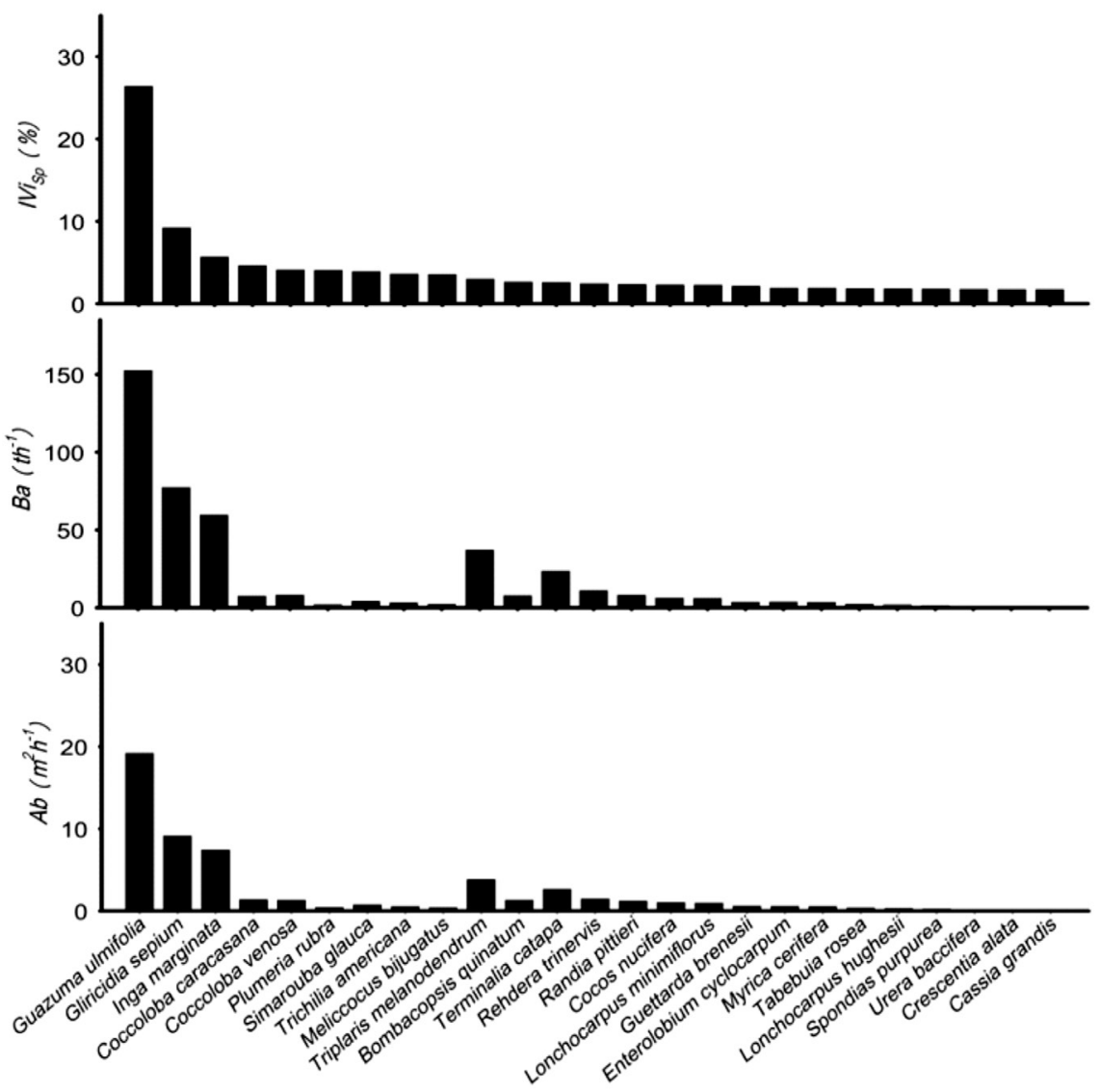

FIG. 3. Índice de valor de importancia (IViSp), biomasa aérea (Ba) y área basal (Ab) para cada una de las especies con hábito arbóreo en Isla San Lucas, Puntarenas, Costa Rica. 


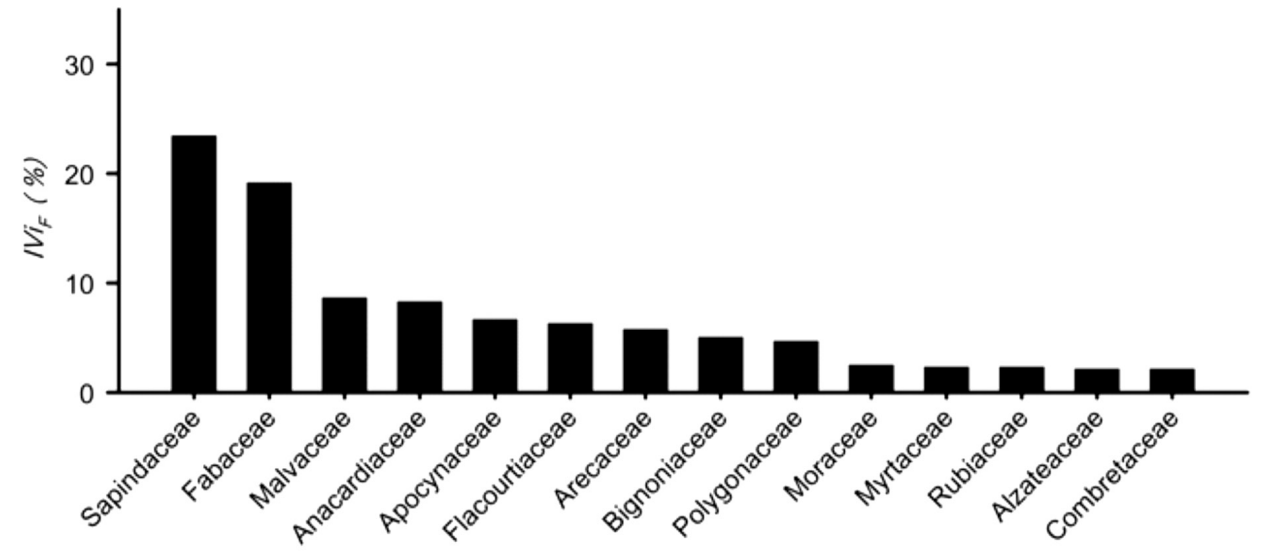

FIG. 4. Índice de valor de importancia (IViF) para cada una de las familias con individuos en estado de latizal en Isla San Lucas, Puntarenas, Costa Rica.

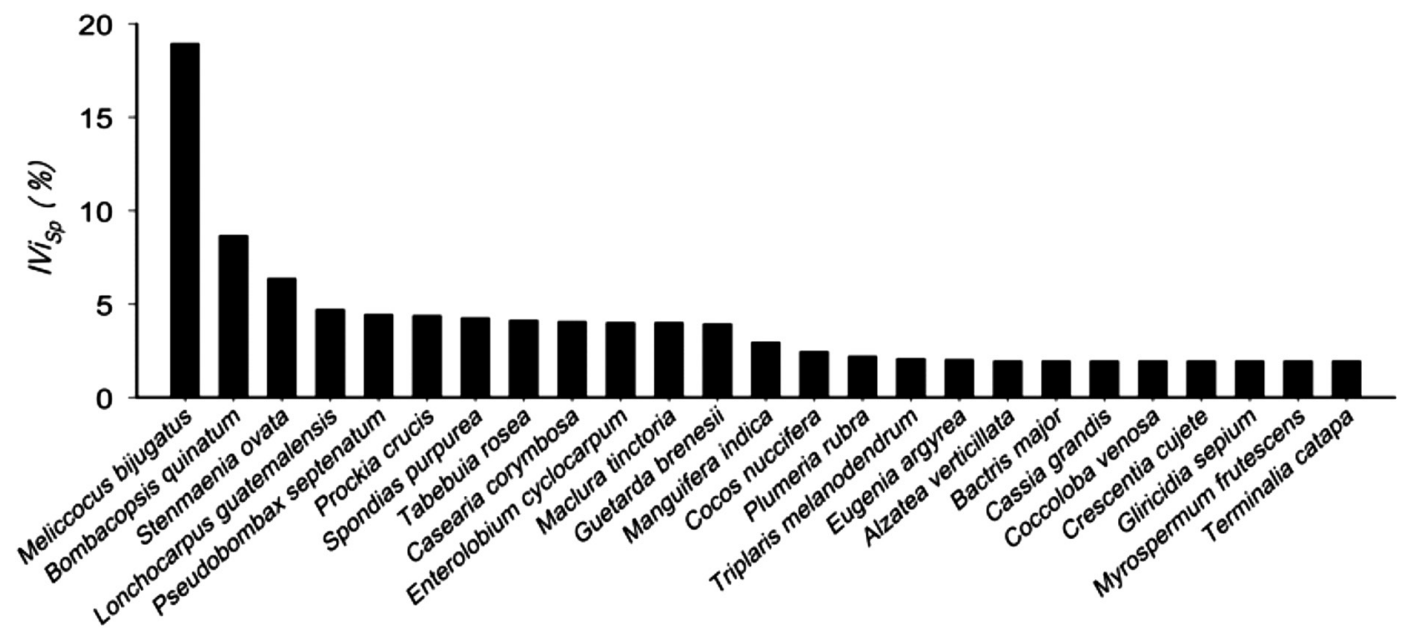

FIG. 5. Índice de valor de importancia (IViF) para cada una de las familias con individuos en estado de latizal en Isla San Lucas, Puntarenas, Costa Rica. 
la comunidad en estado de regeneración ( $n=277, \mathrm{~J}=0,66$, $D=0,22$ ). La similitud de especies fue de 0,52 por lo que la cantidad de especies compartidas entre ambas comunidades vegetales fue de poco más de la mitad de las mismas.

La curva de rarefacción determinó que para una estandarización de la muestra de 51 individuos, la cantidad de especies encontradas para la comunidad de árboles y latizales fue de 20,7 y 13,5 respectivamente (Fig. 6).

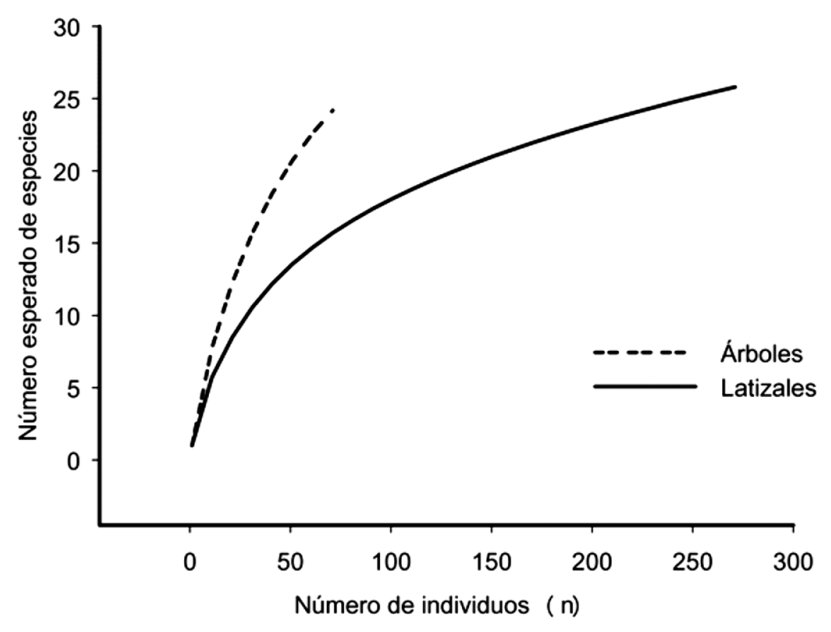

FIG. 6. Curva de rarefacción de la diversidad de árboles y latizales en Isla San Lucas, Puntarenas, Costa Rica.

\section{DISCUSIÓN}

El hecho que el sitio de estudio presentara una cobertura variable y que en promedio fuera no muy sombreado, se debe a que es un ecosistema de bosque secundario, el cual se caracteriza por la presencia de doseles poco cerrados y con cierta entrada de luz (Vieira \& Scariot, 2006), a diferencia de los bosques primarios en los cuales el dosel se estratifica de manera compleja y se va cerrando en la copa lo que crea un sotobosque amplio (Ellsworth \& Reich, 1993). Lo cual, quedó evidenciado con el resultado de la determinación del área basal, que al tener valores relativamente bajos concuerda por lo encontrado en estudios anteriores para bosques secundarios entre 11 y 25 años (Yepes et al., 2010). Con una distribución de J invertida para las clases diamétricas, lo que concuerda con lo encontrado por Ruiz y Fandiño (2007), donde se encontró dicha distribución independientemente del estado sucesional evaluado. Sin embargo otros estudios acotan que la distribución en forma de J invertida es propia de bosques sin señales de perturbaciones, en donde la forma de dicha distribución significa la mejor posibilidad para que ocurra sobreviva una comunidad vegetal (Lamprecht, 1990; Olivier \& Larson, 1990). Las alturas encontradas reflejaron un bosque con poca estratificación y un dosel bajo (entre 5,9 y 9,9 m), característica común para los bosques tropicales secos, máxime si se comparan con bosques tropicales lluviosos los cuales evidencian una mayor complejidad estructural (Murphy \& Lugo, 1986).

La diversidad de especies en el bosque tropical seco normalmente suele ser mucho menor en comparación con bosques tropicales lluviosos (Murphy \& Lugo, 1986). Esto se debe principalmente a las altas temperaturas y la marcada estacionalidad de las lluvias (Mooney, Bullock \& Medina, 1995). En el caso de la isla San Lucas se le suma el hecho de ser una isla de origen marino la cual debió ser colonizada y también el hecho de la influencia de la salinidad en sus aguas subterráneas, por lo que las especies de plantas presentes deben poseer la adaptabilidad ante estas situaciones. La familia Fabaceae obtuvo el mayor IVi con respecto a las demás familias. Esto concuerda con lo encontrado por Gillespie et al. (2000), donde esa misma familia alcanzó junto con Bignoniaceae la mayor diversidad de especies, y también logró el mayor número de individuos tanto para árboles como para arbustos observándose una marcada dominancia. Además Kalacska et al. (2004) encontraron que Fabaceae obtuvo los porcentajes de composición más altos para los estados de sucesión tardíos y intermedios y ocupó la segunda posición para el estado sucesión temprano. En términos de especies, Gliricidia sepium presentó el segundo valor en $A b, B a$ e $I V i$, lo que podría indicar una importancia de la misma como especie pionera y regeneradora del ecosistema. Lo anterior concuerda con lo encontrado por Leiva, Rocha, Mata y Gutiérrez (2009), donde dicha especie se encontró en todos los estados de regeneración excepto en el que poseía una edad mayor a 100 años. Asimismo el número de individuos disminuyo en estados sucesionales de 40 y 60 años y obtuvo una mayor cantidad en aquellos que poseían 10 , 15 y 20 años de edad.

La familia Malvaceae obtuvo la mayoría del porcentaje de su IVi de Guazuma ulmifolia. Según (Sabogal, 1992), la especie Guazuma ulmifolia presentó un IVi de 28,5 en un bosque tropical seco en sucesión de aproximadamente 12 años en Nicaragua. Lo anterior ratifica lo encontrado en nuestro estudio, ya que G. ulmifoila obtuvo un IVi similar, lo que ejemplifica un patrón de regeneración 
constante para dicha especie en este tipo de ecosistema. Lo que confirma lo encontrado previamente respecto a G. ulmifolia como una especie de sucesión temprana, además de que puede establecerse después del fuego (Allen, Allen, Egerton-Warburton, Corkidi \& Gómez-Pompa, 2003; Gordon, Hawthorne, Reyes-García, Sandoval \& Barrance, 2004). Lo anterior sugiere que su alto IVi viene a ser una característica más del bosque tropical seco en sucesión temprana, y con algún tipo de perturbación específica como ocurrió en RVSSL durante mucho tiempo (fue centro penitenciario y sus bosques estuvieron reducidos a pastizales en su mayoría).

En lo respecta a la regeneración, Sapindaceae fue la familia que destacó con valores más altos de $A b, B a$ e IVi. Esto podría ser apoyado con lo dicho por (Gentry, 1995), donde menciona a dicha familia como de gran importancia junto con Rubiaceae, Euphorbiaceae, Flacourtiaceae y Capparidaceae e incluso indica que después de Fabaceae y Bignoniaceae, las familias Rubiaceae y Sapindaceae son la tercer y cuarta familia respectivamente, con mayor importancia en BTS. Sin embargo la representación de Sapindaceae estuvo a cargo únicamente de M. bijugatus, una especie con bajos reportes de abundancia o presencia en otros estudios (Weaver \& Chinea, 2003; Oatham \& Boodram, 2006; Molina \& Lugo, 2006; Ruiz \& Fandiño, 2007) Para los individuos en regeneración se observó la presencia de especies de sucesión tardía tales como B. quinatum, Pseudobombax septenatum (Malvaceae) y E. cyclocarpum, lo cual sugiere que a partir de los remanentes de los antiguos bosques de la isla, se está originando el proceso de regeneración del ecosistema.

Con respecto a la diversidad de especies en cada grupo etario, se logró observar que a una estandarización de 51 individuos, la cantidad de especies encontradas fue menor para latizales, pero esto no se mantuvo para el total de individuos muestreados, donde cada comunidad vegetal obtuvo la misma cantidad de especies (se debe tomar en cuenta que el estado de regeneración no fue evaluado en todas las parcelas establecidas). Esto concuerda a lo encontrado por Leiva et al. (2009), donde se compara la diversidad de latizales contra la de árboles en distintos estados de sucesión y se obtuvo una mayor diversidad de latizales que se encontraban en una sucesión de 10, 15 y 20 años.

La dominancia en latizales estuvo mayormente representada por M. bijugatus, ya que ésta especie presenta una ventaja competitiva debido tanto a la alta producción de frutos así como a la facilidad de dispersión de sus semillas, las cuales podrían ser una fuente de alimento para distintos grupos de animales tales monos aulladores y murciélagos (Flores-Martínez, Ortega \& Ibarra-Manríquez, 2000;
Genoways, Kwiecinski, Larsen, Pedersen \& Larsen, 2010), como el Artibeus jamaicensis presente en la isla. Además, Ortega \& Castro-Arellano (2001), encontraron grupos de semillas y plántulas de varias especies, entre las cuales se encontraba M. bijugatus; ésto como posible evidencia de la dispersión por parte de los murciélagos.

La alta equidad en los árboles se puede deber a la permanencia de individuos remanentes, los cuales eventualmente son aprovechados por aves residentes o migratorias, que a su vez se encargan de dispersar las semillas, ya sea a largas distancias o dejándolas caer cerca de los árboles progenitores, con lo cual se acumulan e incluso podrían formar zonas núcleo de árboles remanentes (Guevara, Purata \& Van der Maarel, 986). La similitud intermedia encontrada entre las clases etarias puede deberse a los remanentes de bosque de la isla, y por ende a la presencia de árboles que aportan a la regeneración contribuyendo de ésta forma a la similitud de especies (Guevara et al., 1986; Harvey \& Haber, 1999). También la aparición de especies de sucesión temprana como G. ulmifolia y $S$. obovata contribuye a la disimilitud (Sabogal, 1992; Allen et al., 2003). Es importante destacar que la isla fue sometida a perturbaciones antropogénicas durante mucho tiempo, tales como tala de árboles y arbustos para distintos fines, quema de pastizales, entre otras, por lo que, es díficil determinar la complicidad del hombre en la función y estructura del bosque y como dicha interferencia influye en la posterior evaluación del mismo mediante índices de diversidad como fue el caso.

Se concluye que el bosque presente en el Refugio Nacional de Vida Silvestre Isla San Lucas, es un bosque secundario joven (10-20 años), esto por sus características estructurales y la composición de especies arbóreas. También cabe destacar que está en procesos de regeneración y es necesario el estudio de una especie como M. bijugatus. La cual está resultando como una especie dominante en el bosque de la isla, contrario a lo reportado anteriormente para el bosque tropical seco 8 (Molina \& Lugo, 2006). Se destaca a la isla San Lucas como un lugar idóneo para estudios de ecología vegetal del bosque seco.

\section{AGRADECIMIENTOS}

AI MINAET y personal institucional por el aval de realizar la investigación en el RNVSISL. A Iván Sandoval Hernández y Alejandro Durán Apuy por los consejos y aportes metodológicos durante la realización del proyecto. A la clase del 2011 de los cursos de Ecología de Poblaciones y Fauna de la Escuela de Ciencias Biológicas de la Universidad Nacional por la toma de datos en el campo. 


\section{REFERENCIAS}

Allen, E.B., Allen, M.F., Egerton-Warburton, L., Corkidi, L., \& Gómez-Pompa, A. (2003). Impacts of Early- and Late-seral Mycorrhizae During Restoration in Seasonal Tropical Forest, Mexico. Ecological Applications, 13, 1701-1717. doi: 10.1890/02-5309.

Bolaños, R., Watsom, V., \& Tosi, J. (2005). Mapa ecológico de Costa Rica (Zonas de Vida), según el sistema de clasificación de zonas de vida del mundo de L.R. Holdrige. Centro Científico Tropical, San José, Costa Rica.

Curtis, J.T., \& McIntosh, R.P. (1950). The Interrelations of Certain Analytic and Synthetic Phytosociological Characters. Ecology, 31(3), 434-455.

Curtis, J.T., \& Mclntosh, R.P. (1951). An Upland Forest Continuum in the Prairie-Forest Border Region of Wisconsin. Ecology, 32(3), 476-496.

Ellsworth, D.S., \& Reich, P.B. (1993). Canopy structure and vertical patterns of photosynthesis and related leaf traits in a deciduous forest. Oecologia, 96(2), 169-178. doi: 10.1007/ BF00317729.

Flores-Martínez, J.J., Ortega, J., \& Ibarra-Manríquez, G. (2000). El hábito alimentario del murciélago zapotero (Artibeus jamaicensis) en Yucatán. Revista Mexicana de Mastozoología, 4, 22-39.

Genoways, H.H., Kwiecinski, G.G., Larsen, P.A., Pedersen, S.C., \& Larsen, R.J. (2010). Bats of the Grenadine Islands, West Indies, and placement of Koopman's Line. Chiroptera Neotropical, 16(1), 501-521.

Gentry, A.H. (1995). Diversity and floristic composition of neotropical dry forests. In S.H. Bullock, H.A. Mooney, \& E. Medina, Seasonally dry tropical forests (pp. 146-194). Cambridge: Cambridge University Press.

Gillespie, T.W., Grijalva, A., \& Farris, C.N. (2000). Diversity composition and structure of tropical dry forests in Central America. Plant Ecology, 147, 37-47. doi: 10.1023/A:1009848525399.

Gordon, J.E., Hawthorne, W.D., Reyes-García, A., Sandoval, G., \& Barrance, A.J. (2004). Assessing landscapes: a case study of tree and shrub diversity in the seasonally dry tropical forests of Oaxaca, Mexico and southern Honduras. Biological Conservation, 117(4), 429-442. doi: 10.1016/j. biocon.2003.08.011.

Guevara, S., Purata, S.E., \& Van der Maarel, E. (1986). The Role of Remnant Forest Trees in Tropical Secondary Succession. Vegetatio, 66(2), 77-84.

Harvey, C.A., \& Haber, W.A. (1999). Remnant trees and the conservation of biodiversity in Costa Rican pastures. Agroforestry Systems, 44, 37-68. doi: 10.1023/A:1006122211692.

Herrerías-Diego, Y., Quesada, M., Stoner, K.E., \& Lobo, J.A. (2006). Effects of Forest Frangmentation on Phenological Patterns and Reproductive Success of the Tropical Dry Forest Tree Ceiba aesculifolia. Conservation Biology, 20(4), 11111120. doi: 10.1111/j.1523-1739.2006.00370.x.
Holdridge, L.R. (1978). Ecología basada en zonas de vida. San José, Costa Rica: Instituto Interamericano de Ciencias Agrícolas.

Janzen, D.H. (1988). Management of Habitat Fragments in a Tropical Dry Forest: Growth. Annals of the Missouri Botanical Garden, 75(1), 105-116.

Kalacska, M., Sanchez-Azofeifa, G.A., Calvo-Alvarado, J.C., Quesada, M., Rivard, B., \& Janzen, D.H. (2004). Species composition, similarity and diversity in three successional stages of a seasonally dry tropical forest. Forest Ecology and Management, 200(1-3), 227-247. doi: 10.1016/j. foreco.2004.07.001.

Kennard, D.K., Gould, K., Putz, F.E., Fredericksen, T.S., \& Morales, F. (2002). Effect of disturbance intensity on regeneration mechanisms in a tropical dry forest. Forest Ecology and Managament, 162(2-3), 197-208. doi: 10.1016/ S0378-1127(01)00506-0.

Lamprecht, H. (1990). Silvicultura en los trópicos: Los ecosistemas forestales en los bosques tropicales y sus especies arbóreasposibilidades y métodos para un aprovechamiento sostenido. Eschborn, Alemania: (GTZ) GMBH.

Lebrija-Trejos, E., Bongers, F., Pérez-García, E.A., \& Meave, J.A. (2008). Successional Change and Resilience of a Very Dry Tropical Deciduous Forest Following Shifting Agriculture. Biotropica, 40(4), 422-431. doi: 10.1111/j.1744-7429.2008.00398.x.

Leiva, J.A., Rocha, O.J., Mata, R., \& Gutiérrez, M.V. (2009). Cronología de la regeneración del bosque tropical seco en Santa Rosa, Guanacaste, Costa Rica. II. La vegetación en relación con el suelo. Revista de Biología Tropical, 57(3), 817-836.

Miller, P.M., \& Kauffman, J.B. (1988). Effects of slash and burn agriculture on species abundance and composition of a tropical deciduous forest. Forest Ecology and Management, 103(2-3), 191-201.

Molina, S., \& Lugo, A.E. (2006). Recovery of a Subtropical Dry Forest After Abandonment of Different Land Uses. Biotropica, 38(3), 354-364. doi: 10.1111/j.1744-7429.2006.00159.x.

Mooney, H.A., Bullock, S.H., \& Medina, E. (1995). Introduction. In S.H. Bullock, H.A. Mooney, \& Medina, E. Seasonally dry tropical forests (pp. 1-8). Cambridge: Cambridge University Press.

Mori, S.A., Boom, B.M., de Carvalho, A.M., \& dos Santos, T.S. (1983). Southern Bahian moist forests. The Botanical Review, 49(2), 155-232.

Murphy, P.G., \& Lugo, A.E. (1986). Ecology of Tropical Dry Forest. Annual Review of Ecology and Systematics, 17, 67-88.

Nebel, G., Kvist, L.P., Vanclay, J.K., Christensen, H., Freitas, L., \& Ruíz, J. (2001). Structure and floristic composition of flood plain forests in the Peruvian Amazon: I. Overstorey. Forest Ecology and Management, 150(1-2), 27-57. doi: 10.1016/ S0378-1127(00)00680-0.

Oatham, M.P., \& Boodram, N. (2006). The dry forest vegetation communities of little Tobago Island, West Indies: floristic affinities. Tropical Ecology, 47(2), 211-228. 
Ortega, J., \& Castro-Arellano, I. (2001). Artibeus jamaicensis. Mammalian Species, 662: 1-9.

Olivier, C., \& Larson, B.C. (1990). Forest stand dynamics. New York, USA: McGraw-Hill.

Pennington, R.T., Prado, D.E., \& Pendry, C.A. (2000). Neotropical seasonally dry forests and Quaternary vegetation changes. Journal of Biogeography, 27(2), 261-273.

Ruiz, J., \& Fandiño, M.C. (2007). Plantas leñosas del bosque seco tropical de la Isla de Providencia, Colombia, Caribe Sur Occidental. Biota Colombiana, 8(1), 87-98.

Sabogal, C. (1992). Regeneration of tropical dry forests in Central America, with examples from Nicaragua. Journal of Vegetation Science, 3(3), 407-416. doi: 10.2307/3235767.
Vieira, D.L., \& Scariot, A. (2006). Principles of Natural Regeneration of Tropical Dry Forests for Restoration. Restoration Ecology, 14(1), 11-20. doi: 10.1111/j.1526-100X.2006.00100.x.

Weaver, P.L., \& Chinea, J.D. (2003). Secondary Subtropical Dry Forest at the La Tinaja Tract of the Cartagena Lagoon National Wildlife Refuge, Puerto Rico. Caribbean Journal of Science, 39(3), 273-285.

Yepes, A.P., del Valle, J.I., Jaramillo, S.L., \& Orrego, S.A. (2010). Recuperación estructural en bosques sucesionales andinos de Porce (Antioquia, Colombia). Revista de Biología Tropical, 58(1), 427-445. 
